\title{
Effect of 24-month physical activity on cognitive frailty and the role of inflammation: the LIFE randomized clinical trial
}

Zuyun Liu ${ }^{1 *}$, Fang-Chi Hsu², Andrea Trombetti ${ }^{3,4}$, Abby C. King ${ }^{5}$, Christine K. Liu ${ }^{4,6}$, Todd M. Manini ${ }^{7}$, Roger A. Fielding ${ }^{4}$, Marco Pahor ${ }^{7}$, Anne B. Newman ${ }^{8}$, Stephen Kritchevsky ${ }^{9}$, Thomas M. Gill ${ }^{1 *}$ and for the LIFE Study investigators

\begin{abstract}
Background: Whether physical activity can reduce cognitive frailty—a relatively new "compound" phenotype proposed in 2013-and whether the effect of physical activity differs based on levels of inflammation are unknown. Therefore, this study aimed to evaluate the effect of physical activity on cognitive frailty and whether baseline interleukin-6 (IL-6) levels modified this effect.

Methods: We used data from the Lifestyle Interventions and Independence for Elders (LIFE) Study, a multicenter, single-blinded randomized trial conducted at eight US field centers between February 2010 and December 2013. The main outcome was cognitive frailty at 24 months, expressed as an ordinal variable based on the six combinations of its two components: frailty (non-frail, pre-frail, and frail) and mild cognitive impairment (yes, no). Frailty and cognition were assessed by the Study of Osteoporotic Fractures (SOF) index and the Modified Mini-Mental State Examination (3MSE) scale, respectively. Plasma IL-6 was measured at baseline. Of the 1635 original randomized sedentary participants (70-89 years), this study included 1298 participants with data on both cognitive frailty and IL-6 assessments at baseline.

Results: After adjusting for field center, sex, and baseline levels of cognitive frailty, the ordinal logistic regression model revealed that participants in the physical activity group had 21\% lower odds (odds ratio, $0.79 ; 95 \%$ confidence interval, 0.64-0.98) of worsening cognitive frailty over 24 months than those in the health education group. The effect of physical activity on cognitive frailty did not differ according to baseline IL-6 levels ( $P$ for interaction $=0.919$ ). The results did not change after additional adjustment for IL-6 subgroups and the inverse probability of remaining in the study. Comparable results were observed according to age, sex, ethnicity/race, and short physical performance battery score ( $P$ for interaction $=0.835,0.536,0.934$, and 0.458 , respectively).
\end{abstract}

Conclusions: A 24-month structured, moderate-intensity physical activity program reduced cognitive frailty compared with a health education program in sedentary older persons, and this beneficial effect did not differ according to baseline levels of inflammatory biomarker IL-6. These findings suggest that the new cognitive frailty construct is modifiable and highlight the potential of targeting cognitive frailty for promoting healthy aging.

Trial registration: Clinicaltrials.gov, NCT01072500

Keywords: Physical activity, Cognitive frailty, Interleukin-6, Older persons

\footnotetext{
* Correspondence: zuyun.liu@yale.edu; thomas.gill@yale.edu

1 Department of Internal Medicine, Yale School of Medicine, New Haven,

CT, USA

Full list of author information is available at the end of the article
}

(c) The Author(s). 2018 Open Access This article is distributed under the terms of the Creative Commons Attribution 4.0 International License (http://creativecommons.org/licenses/by/4.0/), which permits unrestricted use, distribution, and reproduction in any medium, provided you give appropriate credit to the original author(s) and the source, provide a link to the Creative Commons license, and indicate if changes were made. The Creative Commons Public Domain Dedication waiver (http://creativecommons.org/publicdomain/zero/1.0/) applies to the data made available in this article, unless otherwise stated. 


\section{Background}

Cognitive impairment and physical frailty (hereafter referred to as frailty) are two important determinants of an array of adverse health outcomes in older persons that have typically been studied separately as if they were two independent processes. However, recent studies have challenged this traditional view and demonstrated their close interrelationship [1-4], involving chronic inflammation, hormones, nutrition, etc. [3]. Based on these results, a new conceptual construct-cognitive frailty-characterized by the simultaneous presence of both cognitive impairment and frailty, was proposed in 2013 by an international consensus group [5, 6]. Cognitive frailty is more predictive for adverse health outcomes such as functional disability [7-9], low quality of life [7], and mortality [7], than the two individual components, and has become a new target for healthy aging [10].

Many randomized clinical trials have evaluated the effect of interventions such as physical activity on preventing cognitive impairment, but the results have been conflicting [11-14]. In contrast, physical activity is deemed as a promising intervention for reducing frailty, despite limited evidence [15-17]. However, whether physical activity can reduce cognitive frailty, a relatively new "compound" phenotype, over an extended period of time is currently unknown.

From the perspective of putative mechanisms, physical activity has anti-inflammatory properties [18]. Cross-sectional [19-22] and longitudinal studies [23-25] have suggested that an increased inflammatory state plays a key role in the pathogenesis of frailty [26]. Likewise, research has shown links between inflammation and cognitive impairment [27, 28 ]. Recent reviews suggest that inflammation is one possible underlying pathogenetic pathway linking frailty to cognition $[29,30]$. Furthermore, a recent study found that among nondemented older persons with increased inflammation, a cognitive frailty model has a significant additional predictive effect on the risk of disability than the individual components (i.e., frailty, or cognitive impairment) [31]. Therefore, it is plausible that the putative benefit of physical activity on cognitive frailty is more pronounced among older persons who have an increased inflammatory state. If this hypothesis is confirmed, our understanding of the underlying mechanism of cognitive frailty may be advanced and more aggressive programs may be targeted to subpopulations with heightened inflammation.

To address these unanswered questions, we used data from the Lifestyle Interventions and Independence for Elders (LIFE) Study, the largest and longest randomized trial evaluating the benefits of physical activity in older persons $[32,33]$. The primary report of the LIFE Study demonstrated the benefit of a structured physical activity intervention on major mobility disability compared with a health education program among sedentary communitydwelling older persons [32]. The objectives of this current analysis were two-fold: first, to evaluate the effect of physical activity on cognitive frailty; and second, to determine whether inflammatory biomarkers at baseline, particularly interleukin-6 (IL-6), modified the effect of physical activity on cognitive frailty. Among several systemic biomarkers that are indicative of an inflammatory state, IL-6 is deemed as the "cytokine for gerontologists" in terms of its close relationship with aging and chronic morbidity [34, 35]. Furthermore, IL-6 demonstrates consistent associations with frailty $[19,20,22]$ and cognitive impairment $[27,28]$ in older persons.

\section{Methods}

\section{Trial design and participants}

The design, recruitment, baseline characteristics, and main outcomes of the LIFE Study have been published and described in detail elsewhere [32, 33]. Briefly, the LIFE Study, a multicenter, single-blinded randomized trial (clinicaltrials.gov Identifier: NCT01072500) was conducted at eight US field centers (University of Florida, Gainesville and Jacksonville, Florida; Northwestern University, Chicago, Illinois; Pennington Biomedical Research Center, Baton Rouge, Louisiana; University of Pittsburgh, Pittsburgh, Pennsylvania; Stanford University, Stanford, California; Tufts University, Boston, Massachusetts; Wake Forest University, Winston-Salem, North Carolina; and Yale University, New Haven, Connecticut) between February 2010 and December 2013. Men and women aged 70-89 were eligible if they: (a) were sedentary (reported < $20 \mathrm{~min} /$ week in past month performing structured physical activity (i.e., exercise), and $<125 \mathrm{~min} /$ week of moderate physical activity); (b) had functional limitations, as evidenced by a short physical performance battery (SPPB) score 9 or less out of 12 (the SPPB is an integrative measure of balance, gait, and lower extremity strength); (c) could walk $400 \mathrm{~m}$ in $15 \mathrm{~min}$ or less without the help of someone or a walker; and (d) could safely participate in the intervention as determined by medical history, physical exam, and electrocardiography $[32,33]$. Eligible participants had no diagnosis of dementia or significant cognitive impairment based on the Modified Mini-Mental State Examination [36] (3MSE) after accounting for education and race [32, 33]. Informed consent was obtained from all participants. The institutional review boards at all participating sites approved the study protocol. Of the 1635 original randomized participants, 1592 consented to the baseline blood draw, and a sufficient blood sample was successfully collected from 1535 (94\%) participants. In this study, the 1298 participants who had baseline data on both cognitive frailty and IL-6 were included, and the main analyses focused on the subset of 1164 participants $(71.2 \%)$ who also had follow-up data on cognitive frailty (Fig. 1). 


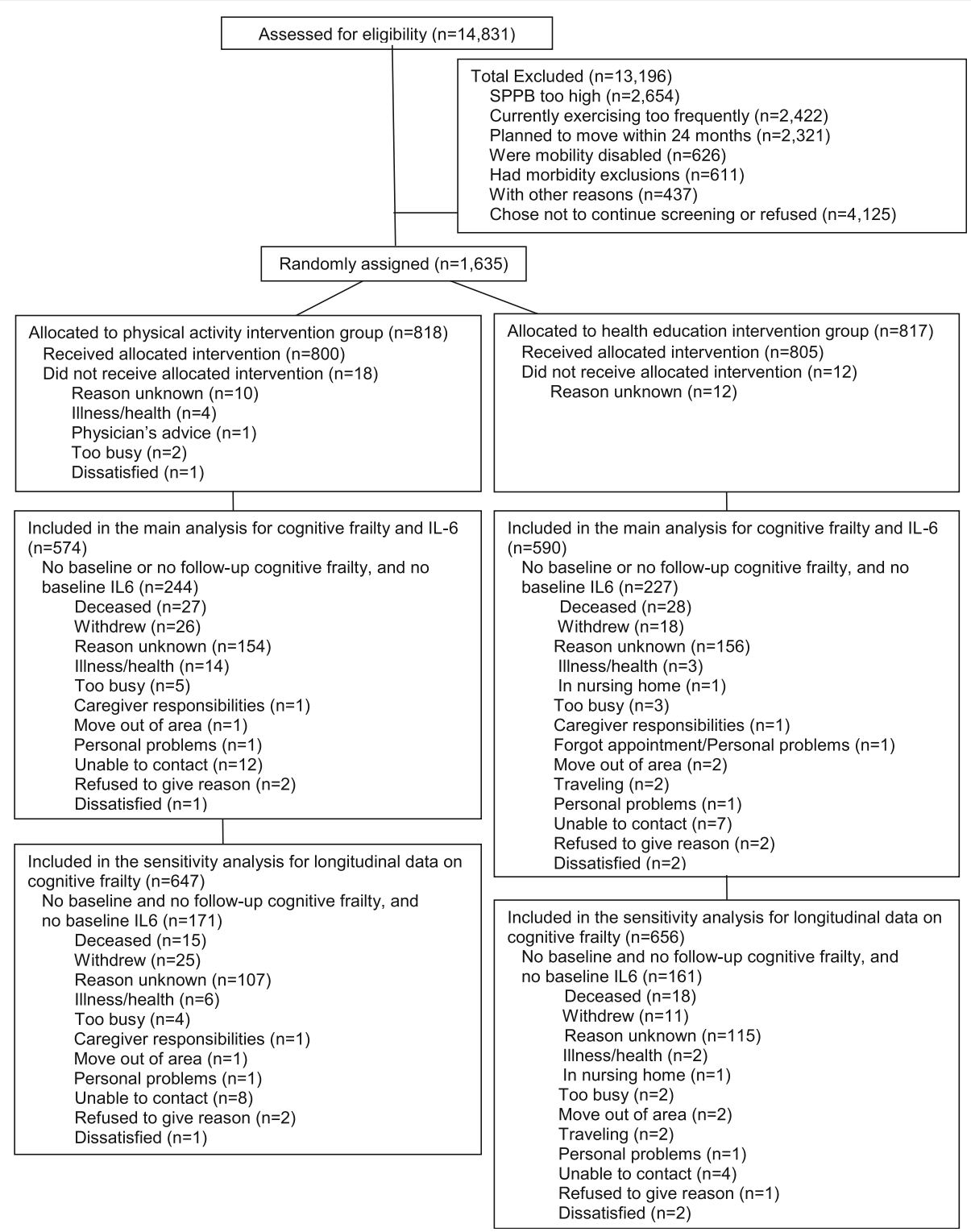

Fig. 1 Flow of study participants through the study. SPPB, Short Physical Performance Battery; IL-6, interleukin-6. The main analysis for cognitive frailty and IL-6 indicates using the ordinal logistic regression models in which baseline levels of cognitive frailty was included as a covariate. The sensitivity analysis for longitudinal data on cognitive frailty indicates using the constrained ordinal logistic regression model in which baseline cognitive frailty was treated as an outcome

\section{Interventions}

Participants were randomly assigned via a secure web-based data management system using a permuted block algorithm (with random block lengths) to either a physical activity intervention or a health education program, stratified by field center and sex. Details about the two interventions are provided elsewhere [32, 33]. Briefly, the physical activity intervention included a goal of $150 \mathrm{~min} /$ week of walking, in addition to strength, flexibility, and balance training. This intervention required attendance at two center-based visits a week and home-based activity three to four times a week. The physical activity sessions were individualized and progressed towards a goal of $30 \mathrm{~min}$ of walking daily at moderate intensity, $10 \mathrm{~min}$ of primarily lower extremity strength training by means of body weight (e.g., chair rises) and ankle weights (2 sets of 10 repetitions), 3$5 \mathrm{~min}$ of large muscle group flexibility exercises, and 10 min of balance training. The participants began with lighter intensity and gradually increased intensity over 
the first 2-3 weeks of the intervention. The Borg's scale of self-perceived exertion [37], with scores ranging from 6 to 20, was used to measure intensity of activity. Participants were asked to walk at an intensity of 13 (activity perception of "somewhat hard"), and perform lower extremity strengthening exercises at an intensity of 15 to 16 (activity perception of "hard").

The health education group attended weekly workshops of health education during the first 26 weeks, and monthly sessions thereafter. Workshops consisted of topics that are relevant to older persons, other than physical activity, such as negotiating the healthcare system, traveling safely, preventive services, and other relevant topics. The program also included a 5- to $10-\mathrm{min}$ instructor-led program of gentle upper extremity stretching or flexibility exercises.

\section{Measurements}

\section{Inflammatory biomarkers-IL-6}

At the baseline visit, blood samples were collected in the early morning (between 7 and 9 a.m.) after a 12-h fast. Blood sampling was postponed (1-2 weeks after recovery of all symptoms) in the event of an acute respiratory, urinary tract, or other infections. All blood was collected, processed, divided into aliquots, and stored locally at $80{ }^{\circ} \mathrm{C}$ until shipment to the Biological Specimen Repository at Wake Forest School of Medicine.

Plasma IL-6 was determined using the Quantikine high-sensitivity enzyme-linked immunosorbent assay kit from R\&D Systems (Minneapolis, MN). All samples were measured in duplicate, and the average of the two values was used for data analyses. We categorized participants into two subgroups according to the median value (i.e., $3.31 \mathrm{pg} / \mathrm{mL}$ ), as described previously [38].

\section{Outcomes assessment SOF frailty}

Frailty status was determined at baseline and 24 months using the Study of Osteoporotic Fractures (SOF) frailty index, which includes three criteria as proposed by Ensrud and colleagues [39]. Self-reported reduced energy level was based on the following question: "During the past week, how often have you felt full of energy". The criterion was present if the participants answered, "Some of the time", "A little bit of the time", or "None of the time". The second criterion, inability to rise from a chair five times without using the arms, was based on the chair rise test from the SPPB. The criterion of weight loss was based on objective measurements and considered as present if the value was $\geq 4.55 \mathrm{~kg}$ or $\geq 5 \%$ during the prior 12 months. Because no objective information on weight loss was available at baseline, this criterion was considered to be present at baseline if the participant answered, "some of the time", or "most of the time" to the question, "How often in the last week did you not feel like eating because your appetite was poor". Participants were considered non-frail if none of the three criteria was present, pre-frail if one was present, and frail if at least two were present [39].

\section{Cognitive assessment}

Cognition was assessed by using the 3MSE scale, which is a 100-point test of global cognitive function. As described previously [40], participants with less than 88 were considered as having mild cognitive impairment (MCI).

\section{Cognitive frailty}

The main outcome was cognitive frailty at 24 months. This new construct has been previously validated [7-9, 41]. To reflect its continuum [42], we created an ordinal variable based on the six combinations of the two components [7-9]: frailty (non-frail, pre-frail, and frail) and $\mathrm{MCI}$ (yes, no). For primary analysis, ordinal variable 1 was created by assigning 0 for no cognitive frailty (i.e., non-frail without MCI), 1 for pre-frail without $\mathrm{MCI}, 2$ for frail without MCI, 3 for non-frail with $\mathrm{MCI}, 4$ for pre-frail with $\mathrm{MCI}$, and 5 for cognitive frailty (i.e., frail with $\mathrm{MCI}$ ). A higher score of this ordinal variable indicates worse cognitive frailty. In the absence of a gold standard, we also created a modified version of this ordinal variable (i.e., ordinal variable 2) for secondary analysis, assigning 2 for non-frail with $\mathrm{MCI}$ and 3 for frail without $\mathrm{MCI}$, while leaving the other values unchanged.

\section{Additional covariates}

Information on covariates was obtained at the baseline visit, including age, sex, education, ethnicity/race, living alone, body mass index, Center for Epidemiology Studies-Depression (CES-D) scores [43], SPPB score $(<8$ or 8-9), and history of hypertension, diabetes, cardiovascular disease, and stroke.

\section{Statistical analyses}

Baseline characteristics were presented by intervention group and baseline IL-6 level using mean (standard deviation [SD]) or numbers (percentages). The distribution of cognitive frailty at baseline and 24 months were presented by intervention group.

To evaluate the effect of physical activity on cognitive frailty, an ordinal logistic regression model was used. This model is written as follows:

$$
\log \operatorname{odds}(Y \leq k)=\alpha_{k}+\beta_{1} X_{1}+\beta_{2} X_{2}+\cdots+\beta_{P} X_{P}
$$

where $k(=1,2,3,4$, or 5$)$ is the value in the outcome measure $Y$ (i.e., cognitive frailty); $X_{i}(i=1$ to $p)$ is the covariate; $\alpha_{k}$ is the intercept respect to $k$; and $\beta_{i}$ is the regression coefficient for each covariate. The covariates included intervention, field center, sex, and baseline levels of 
cognitive frailty (Model 1). To determine whether baseline IL-6 levels modified the effect of physical activity on cognitive frailty, we tested the interaction between intervention and IL-6 subgroups (Model 2). To account for losses to follow-up, we reran this model weighted for the inverse probability of remaining in the study (Model 3 ). For each participant, weights were assigned on the basis of field center, age, sex, education, ethnicity/race, living alone, SPPB score, number of chronic diseases, and 400-m gait speed [32]. Odds ratios (ORs) and their corresponding 95\% confidence intervals (CIs) for the effects of intervention (physical activity vs. health education) and IL-6 subgroups (higher IL-6 vs. lower IL-6) were calculated.

Next, we performed comparisons for the four prespecified subgroups: age (70-79 years vs. $\geq 80$ years), sex (women vs. men), ethnicity/race (White vs. other), and SPPB score $(<8$ vs. $\geq 8)$. The interactions between subgroup and intervention were included in Model 1 to determine whether the intervention effect was the same in each subgroup. In a stratified analysis, we determined the OR for the intervention effect and its $95 \%$ CIs for each subgroup.

To test the robustness of our results, we performed two sensitivity analyses. First, we ran a constrained ordinal logistic regression model incorporating generalized estimating equations, which account for the correlated longitudinal data (e.g., cognitive frailty was measured at baseline and 24 months). We adjusted for field center, sex, visit, and intervention by visit interaction. Baseline cognitive frailty was treated as an outcome, not as a covariate. Second, we reran our models using another cutoff point $(2.5 \mathrm{pg} / \mathrm{ml})$ of IL- 6 as suggested in other studies [38].

In a set of secondary analyses, we repeated the above primary analyses for ordinal variable 2 , and the corresponding results are presented alongside those from primary analyses. We considered two-sided $P$ value $<0.05$ to be statistically significant. Analyses were performed using SAS 9.4 (SAS Institute, Cary, NC).

\section{Results}

On average, the physical activity group attended $63 \%$ of the scheduled sessions (median [interquartile range, IQR], 71\% [50-83\%]), while the health education group attended $73 \%$ of the scheduled sessions (median [IQR], $82 \%$ [63-90\%]) [32]. Table 1 presents the baseline characteristics of participants by intervention group and baseline IL-6 level. The two intervention groups showed similar baseline characteristics. Overall, the mean age was about 79 years, two thirds were women, over $60 \%$ had education beyond high school, about three quarters were White, and nearly one half lived alone. For each intervention group, the two IL-6 subgroups also showed similar baseline characteristics with the following exceptions: the physical activity group had a higher percentage of hypertension in the higher IL-6 subgroup, while the health education group had a lower percentage of women and higher body mass index, and a higher percentage of diabetes in the higher IL-6 subgroup.

Figure 2 shows the distribution of cognitive frailty at baseline and 24 months by intervention group. In the physical activity group, the prevalence of non-frail without MCI and pre-frail without MCI increased from baseline to 24 months, while the prevalence of the other cognitive frailty groups decreased. In the health education group, the prevalence of pre-frail without MCI, frail without $\mathrm{MCI}$, and frail with $\mathrm{MCI}$ increased, whereas the prevalence of non-frail without MCI, non-frail with $\mathrm{MCI}$, and pre-frail with MCI decreased.

Table 2 presents the effect of physical activity on cognitive frailty. In the primary and secondary analyses, participants in the physical activity group had lower odds of worsening cognitive frailty over 24 months than those in the health education group. For example, in the primary analysis, the odds of worsening cognitive frailty was $21 \%$ (OR, 0.79; 95\% CI, 0.64-0.98) lower in the physical activity group compared with the health education group. The effect of physical activity on cognitive frailty did not differ according to baseline IL-6 levels ( $P$ for interaction $=0.919$ for primary analysis and 0.936 for secondary analysis). The results did not change after additional adjustment for IL- 6 subgroups and the inverse probability of remaining in the study (Models 2 and 3). Comparable results were observed according to age, sex, ethnicity/ race, or SPPB score (all $P$ for interaction $>0.05$, Fig. 3).

In sensitivity analyses, we found (1) a significant intervention effect at 24 months for both versions of the cognitive frailty variable in the constrained models (Additional file 1: Table S1), for example, the odds of having worsening cognitive frailty score over 24 months was $20 \%$ (e.g., primary analysis for ordinal variable 1 , OR, 0.80 ; $95 \% \mathrm{CI}, 0.65-0.98$, Model 1) lower in the physical activity group than the health education group; (2) when the alternative cutoff point was used, baseline IL-6 levels did not modify the effect of physical activity on cognitive frailty.

\section{Discussion}

To the best of our knowledge, this is the first study to evaluate the effect of long-term physical activity on cognitive frailty, a new construct in older persons [5]. This study demonstrated that a 24-month structured, mode rate-intensity physical activity program reduced the severity of cognitive frailty compared with a health education program among sedentary older persons and that this benefit was not modified by baseline IL-6.

A recent systematic review with meta-analysis concluded that physical exercise, including moderate intensity aerobic and resistance training, could improve cognitive 


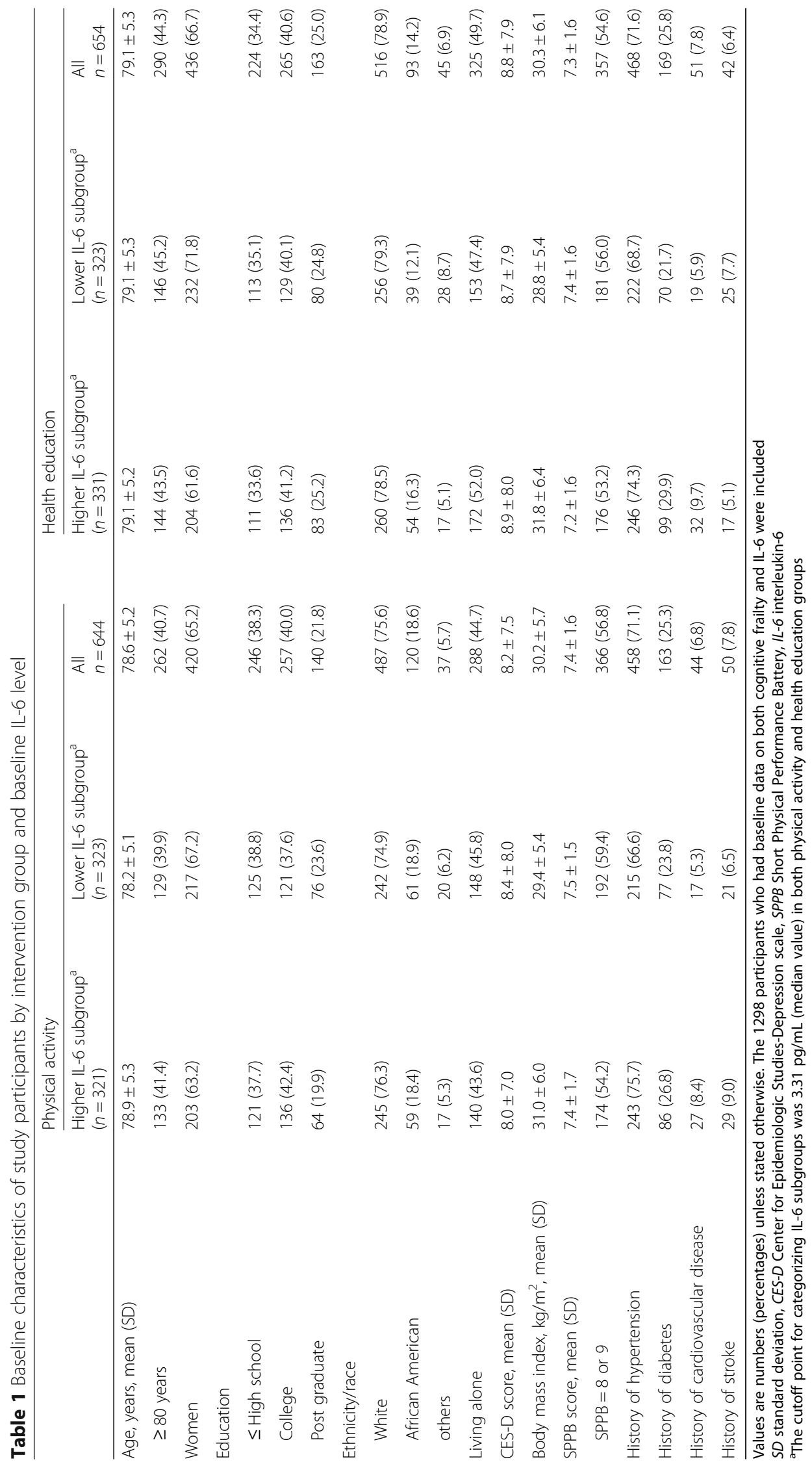




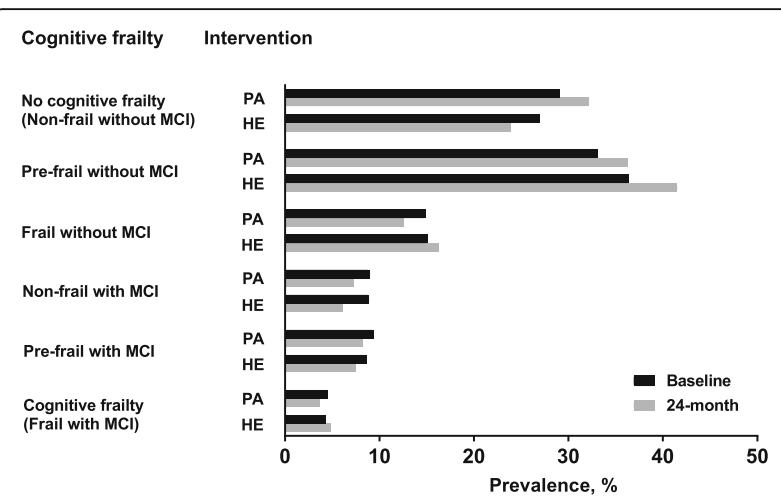

Fig. 2 Distribution of cognitive frailty at baseline and 24 months by intervention group. PA, physical activity; HE, health education. To reflect the continuum of cognitive frailty, we created an ordinal variable based on the six combinations of two components: frailty (non-frail, pre-frail, and frail) and $\mathrm{MCl}$ (yes, no): no cognitive frailty (i.e., non-frail without $\mathrm{MCl}$ ), pre-frail without $\mathrm{MCl}$, frail without $\mathrm{MCl}$, non-frail with $\mathrm{MCl}$, pre-frail with $\mathrm{MCl}$, and cognitive frailty (i.e., frail with $\mathrm{MCl}$ )

function in persons aged 50 years or older, regardless of baseline cognitive status [44]. Another review suggested that multicomponent exercises, including aerobic, resistance, balance, and/or flexibility training, have the most positive effects on cognitive function in healthy older persons over 65 years old [11]. These results are further supported by those described in a systematic review of 32 trials [14]. However, prior results from the LIFE Study showed no difference in global cognition between the two intervention groups [12]. In contrast, interventions such as physical activity have demonstrated relatively consistent positive effects on reversing frailty and/or its components [15-17, 45]. For example, a recent secondary analysis from the LIFE Study found that the 24-month physical activity program was associated with improvement in one criterion (i.e., the inability to rise from a chair five times) of the SOF frailty index [17]. In a recent trial, a 1-year multicomponent exercise intervention including physical activity diminished frailty and improved cognition in frail community-dwelling older persons [46]. Building on these prior results [11-17, 44-46], we provide the first clinical trial evidence for the beneficial effect of physical activity on cognitive frailty in sedentary older persons, suggesting that cognitive frailty may be modifiable and has the potential to serve as a target for promoting healthy aging [10].

We found that baseline IL- 6 levels did not modify the beneficial effect of physical activity on cognitive frailty, indicating that the effect of intervention was not influenced by underlying inflammation. This result suggests that inflammation may not be an important pathophysiological factor of cognitive frailty, despite prior beliefs established on the basis of evidence on cognition and/or frailty [29, 30, 47]. Alternatively, IL-6 may not be the best indicator of inflammation in older persons with functional limitations [48], although it has been widely used in previous studies [34]. Future studies should consider evaluating multiple biomarkers (e.g., tumor necrosis factor- $\alpha$ ) to assess the underlying inflammatory state [49].

The current study has many strengths, including the large sample of an older vulnerable population who were typically excluded in prior randomized trials of physical activity, long duration of intervention and follow-up period, and high retention rate. The availability of IL-6 further provides a unique opportunity to examine the role of inflammation. However, this study also has several limitations. First, the exclusion of participants with dementia or significant cognitive impairment, and those

Table 2 Effect of physical activity on cognitive frailty using ordinal logistic regression models

\begin{tabular}{|c|c|c|c|c|c|c|}
\hline & \multicolumn{2}{|l|}{ Model 1} & \multicolumn{2}{|l|}{ Model 2} & \multicolumn{2}{|l|}{ Model 3} \\
\hline & OR $(95 \% \mathrm{Cl})$ & $P$ value & OR $(95 \% \mathrm{Cl})$ & $P$ value & OR $(95 \% \mathrm{Cl})$ & $P$ value \\
\hline \multicolumn{7}{|l|}{ Primary analysis } \\
\hline Physical activity vs. health education & $0.79(0.64-0.98)$ & 0.032 & $0.79(0.64-0.98)$ & 0.030 & $0.80(0.65-0.97)$ & 0.026 \\
\hline Higher IL-6 vs. lower IL-6 & - & - & $1.11(0.89-1.37)$ & 0.357 & $1.09(0.89-1.33)$ & 0.400 \\
\hline \multicolumn{7}{|l|}{ Secondary analysis } \\
\hline Physical activity vs. health education & $0.77(0.62-0.95)$ & 0.015 & $0.77(0.62-0.95)$ & 0.014 & $0.77(0.63-0.95)$ & 0.012 \\
\hline Higher IL-6 vs. lower IL-6 & - & - & $1.13(0.91-1.40)$ & 0.260 & $1.11(0.91-1.36)$ & 0.301 \\
\hline
\end{tabular}

The 1164 participants who had baseline data on cognitive frailty and IL-6 and follow-up data on cognitive frailty were included OR odds ratio, Cl confidence interval, IL-6 interleukin-6

Ordinal logistic regression was used as described in the Method section. For the primary analysis, ordinal variable 1 was created by assigning 0 for no cognitive frailty (i.e., non-frail without mild cognitive impairment $[\mathrm{MCl}]), 1$ for pre-frail without $\mathrm{MCl}, 2$ for frail without $\mathrm{MCl}, 3$ for non-frail with $\mathrm{MCl}, 4$ for pre-frail with $\mathrm{MCl}$, and 5 for cognitive frailty (i.e., frail with $\mathrm{MCl}$ ). For the secondary analysis, ordinal variable 2 was created by assigning 2 for non-frail with $\mathrm{MCl}$ and 3 for frail without $\mathrm{MCl}$ while the other values remained unchanged

Model 1 adjusted for intervention, field center, sex, and baseline levels for cognitive frailty

As the interaction between intervention groups and IL-6 subgroups was not statistically significant $(P$ for interaction $=0.919$ for primary analysis and 0.936 for secondary analysis), it was not included in Model 2

Model 3 adjusted for the same covariates as in Model 2 but weighted for the inverse probability of remaining in the study 


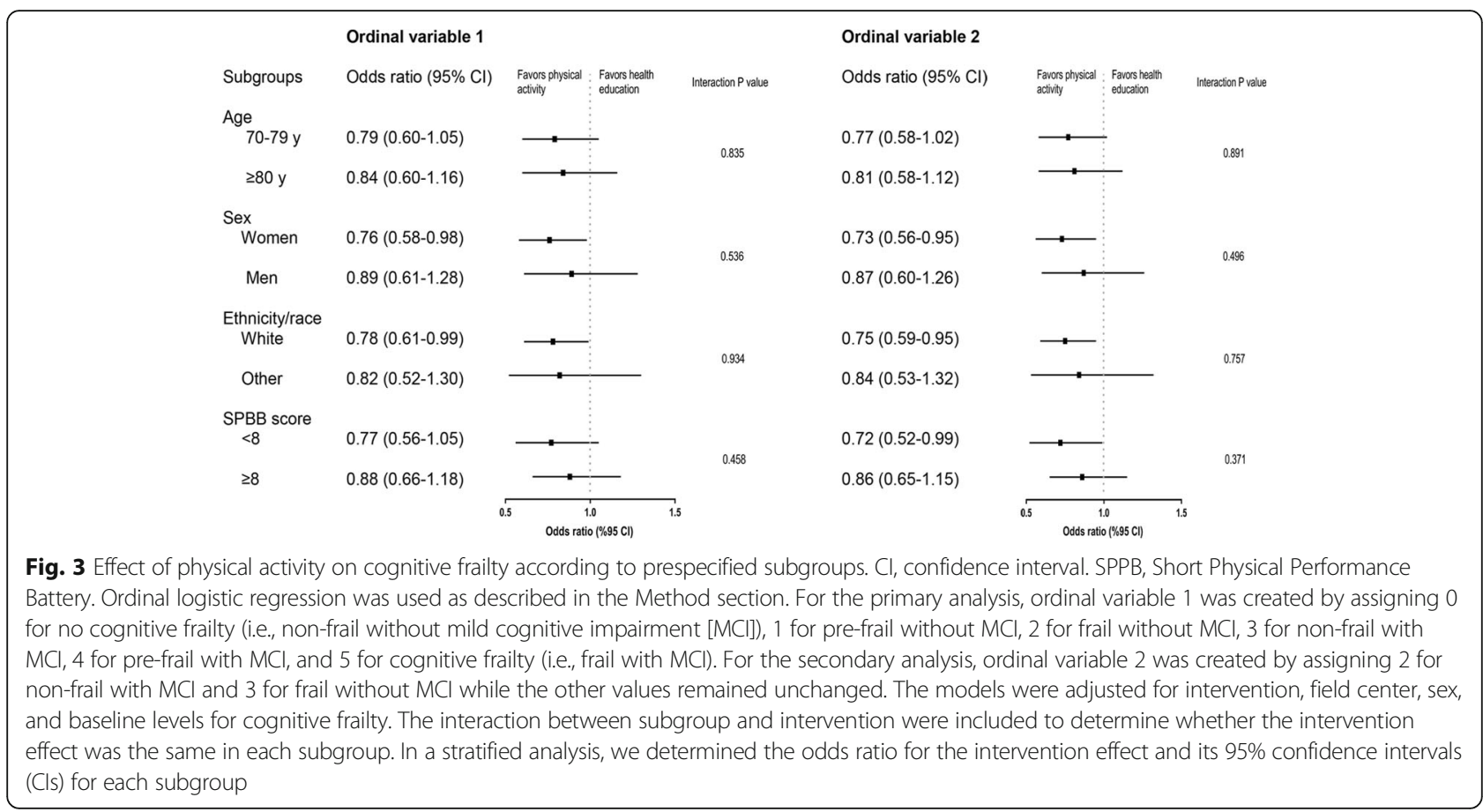

without functional limitation and/or IL-6, may yield a biased sample, reducing the generalizability of the findings. Second, cognitive frailty was neither an entry criterion nor a randomization stratum since this was a secondary analysis that was not pre-specified in the original protocol. Third, because information was not available on weight loss at baseline, loss of appetite was substituted as one of the frailty criteria [50]. Fourth, although frailty and MCI may affect health to differing degrees, each of the cognitive frailty groups was weighted equally when creating the ordinal variable. Fifth, power may not have been adequate to detect a modifying effect of IL-6, which was also not pre-specified; therefore, future studies with larger sample size are warranted. Finally, the physical activity intervention included a coordinated program of endurance, strength, flexibility, and balance training, making it difficult to formally separate which components of the intervention were effective in reducing cognitive frailty.

\section{Conclusions}

Compared with a health education program, a structured, moderate-intensity physical activity program reduced the severity of cognitive frailty over 2 years among sedentary older persons, but this beneficial effect did not differ according to the baseline levels of inflammatory biomarker IL-6. These findings suggest that the new cognitive frailty construct is modifiable and highlight the potential of targeting cognitive frailty for promoting healthy aging.

\section{Additional files}

Additional file 1: Table S1. Effect of physical activity on cognitive frailty using constrained ordinal logistic regression models. (DOCX 29 kb)

Additional file 2: Research investigators for the LIFE Study. (DOCX 15 kb)

\section{Abbreviations}

3MSE: Modified Mini-Mental State Examination; CES-D: Center for Epidemiology Studies-Depression; Cl: Confidence interval; IL-6: Interleukin-6; LIFE: Lifestyle Interventions and Independence for Elders; MCl: Mild cognitive impairment; OR: Odds ratio; SD: Standard deviation; SOF: Study of Osteoporotic Fractures; SPPB: Short Physical Performance Battery

\section{Acknowledgements}

We thank Dr. Heather Allore for the help with the statistical analyses and writing. A complete listing of the LIFE investigators is provided in Additional file 2.

\section{Funding}

The Lifestyle Interventions and Independence for Elders Study is funded by a National Institutes of Health/National Institute on Aging Cooperative Agreement \#UO1 AG22376 and a supplement from the National Heart, Lung and Blood Institute 3U01AG022376- 05A2S, and sponsored in part by the Intramural Research Program, National Institute on Aging, NIH. The research is partially supported by the Claude D. Pepper Older Americans Independence Centers at the University of Florida (1 P30 AG028740), Wake Forest University (1 P30 AG21332), Tufts University (1P30AG031679), University of Pittsburgh (P30 AG024827), and Yale University (P30AG021342) and the NIH/NCRR CTSA at Stanford University (UL1 RR025744). Tufts University is also supported by the Boston Rehabilitation Outcomes Center (1R24HD065688-01A1). LIFE investigators are also partially supported by the following: Dr. Thomas Gill (Yale University) is the recipient of an Academic Leadership Award (K07AG3587) from the National Institute on Aging. Dr. Carlos Fragoso (Spirometry Reading Center, Yale University) is the recipient of a Career Development Award from the Department of Veterans Affairs. Dr. Roger Fielding (Tufts University) is partially supported by the US Department of Agriculture, under agreement No. 58-1950-0-014. Any opinions, findings, conclusion, or recommendations expressed in this publication are those of the author(s) and do not necessarily reflect the view of the US Dept of Agriculture. Dr. Liu is the recipient of a James Hudson Brown-Alexander B. Coxe 
Fellowship from Yale School of Medicine, and he also received support from U01AG022376.

\section{Availability of data and materials}

The datasets generated and/or analyzed during the current study are available in the LIFE Study repository, https://www.thelifestudy.org/public/index.cfm, and are available from the LIFE Study Investigators on reasonable request.

\section{Authors' contributions}

ZL and TMG had full access to all of the data in the study and take responsibility for the integrity of the data and the accuracy of the data analysis. The statistical code used to generate the results may be obtained from FCH. All authors participated in designing the analyses, interpreting the results, and writing the manuscript. All authors read and approved the final manuscript.

\section{Ethics approval and consent to participate}

The study protocol, available on request at https://www.thelifestudy.org/ public/index.cfm, was approved by the institutional review boards at all participating sites (University of Florida, Gainesville and Jacksonville, Florida [617-2009]; Northwestern University, Chicago, IL [STU00020824]; Pennington Biomedical Research Center, Baton Rouge, Louisiana, LA [PBRC 29033]; University of Pittsburgh, Pittsburgh, PA [PRO09090386]; Stanford University, Stanford, CA [5136 (Panel:7)]; Tufts University, Boston, MA [9261]; Wake Forest University, Winston-Salem, NC [IRB00011584]; and Yale University, New Haven, CT [0911005926]). Written informed consent was obtained from all participants.

\section{Consent for publication}

Not applicable.

\section{Competing interests}

Dr. Fielding reports grants, personal fees. and other from Axcella Health, personal fees from Cytokinetics, grants and personal fees from Biophytis, personal fees from Amazentis, grants and personal fees from Nestle', grants and personal fees from Astellas, personal fees from Glaxo Smithkline, personal fees from Abbott, and grant from Lonza outside the submitted work. The other authors declare that they have no competing interests.

\section{Publisher's Note}

Springer Nature remains neutral with regard to jurisdictional claims in published maps and institutional affiliations.

\section{Author details}

${ }^{1}$ Department of Internal Medicine, Yale School of Medicine, New Haven, CT, USA. ${ }^{2}$ Department of Biostatistical Sciences, Wake Forest University Health Sciences, Winston Salem, NC, USA. ${ }^{3}$ Division of Bone Diseases, Department of Internal Medicine Specialties, Geneva University Hospitals and Faculty of Medicine, Geneva, Switzerland. ${ }^{4}$ Nutrition, Exercise Physiology and Sarcopenia Laboratory, Jean Mayer USDA Human Nutrition Research Center on Aging, Tufts University, Boston, MA, USA. ${ }^{5}$ Department of Health Research and Policy and the Stanford Prevention Research Center, Department of Medicine, Stanford University, School of Medicine, Stanford, CA, USA. '5ection of Geriatrics, Boston University School of Medicine, Boston, MA, USA. ${ }^{7}$ Department of Aging and Geriatric Research, University of Florida, Gainesville, FL, USA. ${ }^{8}$ Department of Epidemiology, Graduate School of Public Health, University of Pittsburgh, Pittsburgh, PA, USA. ${ }^{9}$ Sticht Center on Aging, Wake Forest School of Medicine, Winston Salem, NC, USA.

\section{Received: 22 March 2018 Accepted: 12 September 2018}

\section{Published online: 24 October 2018}

\section{References}

1. Alencar MA, Dias JM, Figueiredo LC, Dias RC. Frailty and cognitive impairment among community-dwelling elderly. Arq Neuropsiquiatr. 2013; 71:362-7.

2. Raji MA, Al Snih S, Ostir GV, Markides KS, Ottenbacher KJ. Cognitive status and future risk of frailty in older Mexican Americans. J Gerontol A Biol Sci Med Sci. 2010;65:1228-34.
3. Robertson DA, Savva GM, Kenny RA. Frailty and cognitive impairment--a review of the evidence and causal mechanisms. Ageing Res Rev. 2013;12:840-51

4. Canevelli M, Cesari M, van Kan GA. Frailty and cognitive decline: how do they relate? Curr Opin Clin Nutr Metab Care. 2015;18:43-50.

5. Kelaiditi E, Cesari M, Canevelli M, van Kan GA, Ousset PJ, Gillette-Guyonnet S, et al. Cognitive frailty: rational and definition from an (I.A.N.A./.A.G.G.) international consensus group. J Nutr Health Aging. 2013;17:726-34.

6. Woods AJ, Cohen RA, Pahor M. Cognitive frailty: frontiers and challenges. J Nutr Health Aging. 2013;17:741-3.

7. Feng L, Zin Nyunt MS, Gao Q, Feng L, Yap KB, Ng TP. Cognitive frailty and adverse health outcomes: findings from the Singapore Longitudinal Ageing Studies (SLAS). J Am Med Dir Assoc. 2016;18:252-8.

8. Shimada H, Makizako H, Lee S, Doi T, Lee S, Tsutsumimoto K, et al. Impact of cognitive frailty on daily activities in older persons. J Nutr Health Aging. 2016:20:729-35

9. Avila-Funes JA, Amieva H, Barberger-Gateau P, Le Goff M, Raoux N, Ritchie K, et al. Cognitive impairment improves the predictive validity of the phenotype of frailty for adverse health outcomes: the three-city study. J Am Geriatr Soc. 2009;57:453-61.

10. Cano A. Cognitive frailty, a new target for healthy ageing. Maturitas. 2015; 82:139-40.

11. Saez de Asteasu ML, Martinez-Velilla N, Zambom-Ferraresi F, Casas-Herrero A, Izquierdo M. Role of physical exercise on cognitive function in healthy older adults: a systematic review of randomized clinical trials. Ageing Res Rev. 2017:37:117-34

12. Sink KM, Espeland MA, Castro CM, Church T, Cohen R, Dodson JA, et al. Effect of a 24-month physical activity intervention vs health education on cognitive outcomes in sedentary older adults: the LIFE Randomized Trial. JAMA. 2015:314:781-90.

13. Williamson JD, Espeland M, Kritchevsky SB, Newman AB, King AC, Pahor M, et al. Changes in cognitive function in a randomized trial of physical activity: results of the lifestyle interventions and independence for elders pilot study. J Gerontol A Biol Sci Med Sci. 2009;64:688-94.

14. Brasure M, Desai P, Davila H, Nelson VA, Calvert C, Jutkowitz E, et al. Physical activity interventions in preventing cognitive decline and Alzheimer-type dementia: a systematic review. Ann Intern Med. 2018;168:30-8.

15. Cesari M, Vellas B, Hsu FC, Newman AB, Doss H, King AC, et al. A physical activity intervention to treat the frailty syndrome in older persons-results from the LIFE-P study. J Gerontol A Biol Sci Med Sci. 2015;70:216-22.

16. de Labra C, Guimaraes-Pinheiro C, Maseda A, Lorenzo T, Millan-Calenti JC. Effects of physical exercise interventions in frail older adults: a systematic review of randomized controlled trials. BMC Geriatr. 2015;15:154.

17. Trombetti A, Hars M, Hsu FC, Reid KF, Church TS, Gill TM, et al. Effect of physical activity on frailty: secondary analysis of a randomized controlled trial. Ann Intern Med. 2018;168:309-16.

18. Pedersen BK. Anti-inflammatory effects of exercise: role in diabetes and cardiovascular disease. Eur J Clin Investig. 2017;47:600-11.

19. Collerton J, Martin-Ruiz C, Davies K, Hilkens CM, Isaacs J, Kolenda C, et al. Frailty and the role of inflammation, immunosenescence and cellular ageing in the very old: cross-sectional findings from the Newcastle $85+$ Study. Mech Ageing Dev. 2012;133:456-66.

20. Darvin K, Randolph A, Ovalles S, Halade D, Breeding L, Richardson A, et al. Plasma protein biomarkers of the geriatric syndrome of frailty. J Gerontol A Biol Sci Med Sci. 2014:69:182-6.

21. Walston J, McBurnie MA, Newman A, Tracy RP, Kop WJ, Hirsch CH, et al. Frailty and activation of the inflammation and coagulation systems with and without clinical comorbidities: results from the Cardiovascular Health Study. Arch Intern Med. 2002;162:2333-41.

22. Schmaltz HN, Fried LP, Xue QL, Walston J, Leng SX, Semba RD. Chronic cytomegalovirus infection and inflammation are associated with prevalent frailty in community-dwelling older women. J Am Geriatr Soc. 2005;53:747-54

23. Barzilay Jl, Blaum C, Moore T, Xue QL, Hirsch CH, Walston JD, et al. Insulin resistance and inflammation as precursors of frailty: the Cardiovascular Health Study. Arch Intern Med. 2007;167:635-41.

24. Puts MT, Visser M, Twisk JW, Deeg DJ, Lips P. Endocrine and inflammatory markers as predictors of frailty. Clin Endocrinol. 2005;63:403-11.

25. Gale CR, Baylis D, Cooper C, Sayer AA. Inflammatory markers and incident frailty in men and women: the English Longitudinal Study of Ageing. Age (Dordr). 2013;35:2493-501. 
26. Yao X, Li H, Leng SX. Inflammation and immune system alterations in frailty. Clin Geriatr Med. 2011;27:79-87.

27. Mooijaart SP, Sattar N, Trompet S, Lucke J, Stott DJ, Ford I, et al. Circulating interleukin-6 concentration and cognitive decline in old age: the PROSPER study. J Intern Med. 2013;274:77-85.

28. Wright CB, Sacco RL, Rundek T, Delman J, Rabbani L, Elkind M. Interleukin-6 is associated with cognitive function: the Northern Manhattan Study. J Stroke Cerebrovasc Dis. 2006;15:34-8.

29. Panza F, Seripa D, Solfrizzi V, Tortelli R, Greco A, Pilotto A, et al. Targeting cognitive frailty: clinical and neurobiological roadmap for a single complex phenotype. J Alzheimers Dis. 2015;47:793-813.

30. Panza F, Solfrizzi V, Barulli MR, Santamato A, Seripa D, Pilotto A, et al. Cognitive frailty: a systematic review of epidemiological and neurobiological evidence of an age-related clinical condition. Rejuvenation Res. 2015;18:389-412.

31. Solfrizzi V, Scafato E, Lozupone M, Seripa D, Giannini M, Sardone R, et al. Additive role of a potentially reversible cognitive frailty model and inflammatory state on the risk of disability: the Italian Longitudinal Study on Aging. Am J Geriatr Psychiatry. 2017;25:1236-48.

32. Pahor M, Guralnik JM, Ambrosius WT, Blair S, Bonds DE, Church TS, et al. Effect of structured physical activity on prevention of major mobility disability in older adults: the LIFE study randomized clinical trial. JAMA. 2014;311:2387-96.

33. Fielding RA, Rejeski WJ, Blair S, Church T, Espeland MA, Gill TM, et al. The lifestyle interventions and independence for elders study: design and methods. J Gerontol A Biol Sci Med Sci. 2011;66:1226-37.

34. Ershler WB. Interleukin-6: a cytokine for gerontologists. J Am Geriatr Soc. 1993:41:176-81.

35. Maggio M, Guralnik JM, Longo DL, Ferrucci L. Interleukin-6 in aging and chronic disease: a magnificent pathway. J Gerontol A Biol Sci Med Sci. 2006; 61:575-84.

36. Teng EL, Chui HC. The modified mini-mental state (3MS) examination. J Clin Psychiatry. 1987;48:314-8.

37. Borg G. Borg's Perceived Exertion and Pain Scales. Champaign: Human Kinetics; 1998.

38. Ferrucci L, Harris TB, Guralnik JM, Tracy RP, Corti MC, Cohen HJ, et al. Serum IL-6 level and the development of disability in older persons. J Am Geriatr Soc. 1999;47:639-46.

39. Ensrud KE, Ewing SK, Taylor BC, Fink HA, Cawthon PM, Stone KL, et al. Comparison of 2 frailty indexes for prediction of falls, disability, fractures, and death in older women. Arch Intern Med. 2008;168:382-9.

40. Sink KM, Espeland MA, Rushing J, Castro CM, Church TS, Cohen R, et al. The LIFE cognition study: design and baseline characteristics. Clin Interv Aging. 2014;9:1425-36.

41. Sargent $L$, Brown R. Assessing the current state of cognitive frailty: measurement properties. J Nutr Health Aging. 2017;21:152-60.

42. Fougere B, Delrieu J, Del Campo N, Soriano G, Sourdet S, Vellas B. Cognitive frailty: mechanisms, tools to measure, prevention and controversy. Clin Geriatr Med. 2017:33:339-55.

43. Weissman MM, Sholomskas D, Pottenger M, Prusoff BA, Locke BZ. Assessing depressive symptoms in five psychiatric populations: a validation study. Am J Epidemiol. 1977;106:203-14.

44. Northey JM, Cherbuin N, Pumpa KL, Smee DJ, Rattray B. Exercise interventions for cognitive function in adults older than 50: a systematic review with meta-analysis. Br J Sports Med. 2018;52:154-60.

45. Luger E, Dorner TE, Haider S, Kapan A, Lackinger C, Schindler K. Effects of a Home-Based and Volunteer-Administered Physical Training, Nutritional, and Social Support Program on Malnutrition and Frailty in Older Persons: A Randomized Controlled Trial. J Am Med Dir Assoc. 2016;17:671 e009-671.e016.

46. Tarazona-Santabalbina FJ, Gomez-Cabrera MC, Perez-Ros P, Martinez-Arnau FM, Cabo H, Tsaparas $\mathrm{K}$, et al. A multicomponent exercise intervention that reverses frailty and improves cognition, emotion, and social networking in the community-dwelling frail elderly: a randomized clinical trial. J Am Med Dir Assoc. 2016;17:426-33.

47. Ruan Q, D'Onofrio G, Sancarlo D, Greco A, Lozupone M, Seripa D, et al. Emerging biomarkers and screening for cognitive frailty. Aging Clin Exp Res. 2017;29:1075-86.

48. Marzetti E, Landi F, Marini F, Cesari M, Buford TW, Manini TM, et al. Patterns of circulating inflammatory biomarkers in older persons with varying levels of physical performance: a partial least squares-discriminant analysis approach. Front Med (Lausanne). 2014;1:27.
49. Roubenoff R. The "cytokine for gerontologists" has some company. J Gerontol A Biol Sci Med Sci. 2014;69:163-4.

50. Solheim TS, Blum D, Fayers PM, Hjermstad MJ, Stene GB, Strasser F, et al. Weight loss, appetite loss and food intake in cancer patients with cancer cachexia: three peas in a pod? - analysis from a multicenter cross sectional study. Acta Oncol. 2014;53:539-46.
Ready to submit your research? Choose BMC and benefit from:

- fast, convenient online submission

- thorough peer review by experienced researchers in your field

- rapid publication on acceptance

- support for research data, including large and complex data types

- gold Open Access which fosters wider collaboration and increased citations

- maximum visibility for your research: over $100 \mathrm{M}$ website views per year

At BMC, research is always in progress.

Learn more biomedcentral.com/submissions 